\title{
As dusk falls: collective decisions about the return to sleeping sites in meerkats
}

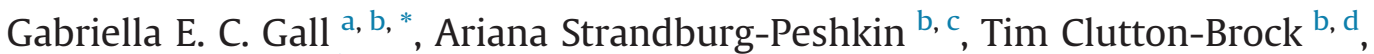 \\ Marta B. Manser ${ }^{\mathrm{a}, \mathrm{b}}$ \\ a Department of Evolutionary Biology and Environmental Studies, University of Zurich, Zurich, Switzerland \\ ${ }^{\mathrm{b}}$ Kalahari Meerkat Project, Kuruman River Reserve, Northern Cape, South Africa \\ ${ }^{\mathrm{c}}$ Department of Migration and Immuno-ecology, Max Planck Institute for Ornithology, Radolfzell, Germany \\ ${ }^{\mathrm{d}}$ Large Animal Research Group, Department of Zoology, University of Cambridge, Cambridge, U.K.
}

Social animal groups often make consensus decisions about when to return to a sleeping site after a day of foraging. These decisions can depend on extrinsic as well as intrinsic factors, and can range from unshared to shared. Here we investigated how decisions of meerkats, Suricata suricatta, to return to their burrows are coordinated, whether they are shared or monopolized by dominant individuals, and what factors influence the timing and speed of return. Individual meerkats can initiate group movements using 'lead' calls, and groups can change foraging patches using 'move' calls in a quorum response. We found that both call types could be produced during the return to the burrow, with the probability of move calls increasing as sunset approached, and the probability of lead calls increasing with greater

Keywords:

decision making

initiation of return

meerkat

termination of activity

time constraints

urgency distance to the burrow when sunset was imminent. Dominant and subordinate individuals did not differ significantly in move and lead call rate. Further, the time of return was better predicted by the foraging success of all subordinates in the group (with the group returning later when success was low) than by the foraging success of the dominant individuals. This suggests that decisions to return are shared rather than controlled by dominants. The speed of return depended both on extrinsic factors, such as the presence of pups, the time until sunset and the distance to the burrow, and on intrinsic factors such as satiation. Our results indicate that both the speed and timing of the return depend on urgency, and the higher incidence of lead calls when groups are far away from the burrow near dark suggests a possible change in the decision process from shared to unshared as urgency increases. Our study highlights the impact of time constraints during decision-making processes and in particular on the level of decision sharing.

Consensus decisions are necessary when individuals within a group must synchronize their activities to maintain the benefits of group living (Krause \& Ruxton, 2002). As group members may differ in their preferences for the type and timing of activities, consensus decisions are typically connected to consensus costs to individuals for whom the decision outcome is suboptimal (Conradt \& Roper, 2003, 2009). Consensus decisions can range from 'shared', when all or many individuals join in the decision-making process, to 'unshared' when only one or a few individuals emerge as leaders (Conradt \& Roper, 2005). Evidence from laboratory experiments

\footnotetext{
* Correspondence: G.E.C. Gall, Department of Evolutionary Biology and Environmental Studies, University of Zurich, Winterthurerstrasse 190, CH-8057 Zurich, Switzerland.
}

E-mail address: gabriella.gall@ieu.uzh.ch (G. E. C. Gall).
(Chittka, Skorupski, \& Raine, 2009; Franks, DechaumeMoncharmont, Hanmore, \& Reynolds, 2009; Franks, Dornhaus, Fitzsimmons, \& Stevens, 2003; Ward, Herbert-Read, Sumpter, \& Krause, 2011) and theoretical models (Sumpter \& Pratt, 2009) shows that collective decision-making processes are greatly affected by time constraints (Conradt, 2011) and can as a consequence shift from highly shared to less shared decisions within the same overall context (Franks et al., 2003). However, evidence from group-living species in their natural environment on how time constraints influence group decisions is currently lacking.

Many social mammal groups in the wild need to reach collective decisions about when to leave on daily foraging trips, where to go, when to return and where to spend each night. Previous analyses have mostly examined the departure for daily foraging trips, and have shown that in some species leadership is determined by the 
social rank of an individual (Boinski, 1993; King, Douglas, Huchard, Isaac, \& Cowlishaw, 2008). Furthermore, energetic demands can drive variation in leadership, with the hungriest individuals being the most likely to initiate group departure (Fischhoff et al., 2007; Furrer, Kunc, \& Manser, 2012; King \& Sueur, 2011). If the needs of an individual are not entirely satisfied after a decision is taken, it can adjust its behaviour by allocating its time differently, for example instead of spending the time vigilant or resting it may forage, thereby reducing consensus costs (Dunbar \& Dunbar, 1988). However, when time is the limiting factor, as for diurnal species when dusk is approaching, individuals are often unable to compensate for unsatisfactory decisions. This can lead to an increase in the consensus costs to each individual and therefore greater conflicts of interest over the timing of a change in group activity. Theoretical models suggest that shared decision processes are likely to evolve in decisions about activity synchronization under a wide variety of circumstances, but particularly when individuals differ strongly in their requirements and consensus costs are high (Conradt \& Roper, 2007, 2009). This is because in contrast to decisions on fixed destinations where individuals must decide on either alternative, group members can compromise and average the timing of decisions, thereby lowering the consensus costs to all individuals (Conradt \& Roper, 2007, 2009). Following this argument further, we predicted that decisions about the timing of an activity would be less shared when conflicts of interest are low and the costs of delaying a decision are high, that is, in situations with time constraints.

Decision making can be investigated on different levels, as individual level processes emerge into group level outcomes. In decisions about the timing of an activity, the change in travel speed or the arrival time at a destination can give insight about the underlying trade-offs and decision processes. For example, the timing of return to a sleeping site is likely to be influenced by the satiation level of group members, with groups returning later when their members are not fully satiated due to low foraging success, and earlier when foraging success was high. Here the differences in foraging success by each member may explain the variation in the type of consensus, namely whether the decision is shared or unshared. Specifically, we expected that if decisions are monopolized by a high-ranking leader, the group would return earlier when this leading individual is fully satiated, independent of the satiation level of its group members. Furthermore, the decision-making process can depend on the 'urgency' of a situation, that is, how fast a decision needs to be taken. House-hunting ants (Leptothorax albipennis) lowered their quorum threshold to decide between multiple nest sites when their previous nest was destroyed, compared to conditions when their old nest site remained intact and the decision time was less of a concern (Franks et al., 2003). When determining the return time to a sleeping site in groups of diurnal species, the urgency of a situation might depend on the distance still to be covered during the remaining daylight time. Groups can adjust their travel speed and the directness of their route towards their target destination, or as with the ant example, they might adjust the decision-making process to save time.

Meerkats, Suricata suricatta, an obligate group-living and social mongoose species, offer an ideal opportunity to study the decisionmaking process underlying the timing of collective returns to sleeping sites. Meerkat groups have a despotic social structure with a dominant pair breeding and subordinate individuals helping to rear the offspring (Clutton-Brock et al., 1998). While meerkats usually change their sleeping burrow every couple of days, they stay at the same burrow for up to 4 weeks when they have very young pups, not yet foraging with the group (Clutton-Brock et al., 1998). When the pups start joining the group on the daily foraging trips, subordinate individuals, in particular, contribute to feeding and guarding them (Clutton-Brock, Brotherton, et al., 2001). In meerkats, a single individual can initiate group departure from the burrow (Turbé, 2006) often mediated through 'lead calls', whereby lead-calling individuals are more frequently followed than individuals that depart silently (Turbé, 2006). When foraging, all individuals can contribute in decisions to change the foraging patch using 'move calls' (Bousquet, Sumpter, \& Manser, 2011). These calls are used in a quorum mechanism, whereby a minimum number of individuals must be in favour of a change in activity or destination in order to be followed by the rest of the group (Bousquet et al., 2011; Ward, Sumpter, Couzin, Hart, \& Krause, 2008). Move and lead calls are part of a continuous graded call system with move calls on one end and lead calls on the other end of the acoustic spectrum (Kalahari Meerkat Project, 2017) and both call types have been observed to be produced in the evening shortly before the return of a group to its sleeping site (G.E.C. Gall \& M. B. Manser, personal observation). Groups can return to their sleeping burrow in two ways, which can be broadly categorized as slow (foraging until they reach their sleeping burrow) or fast (interrupting their foraging to run back to a sleeping burrow, often producing move and lead calls; G.E.C. Gall \& M. B. Manser, personal observation).

Few studies have explored the decisions groups have to make at the end of each foraging day, and those that have focused on the selection of sleeping sites (Fleischmann \& Kerth, 2014; Huang, Wei, Li, Li, \& Sun, 2003). Although collective returns represent critical consensus decisions that occur daily in the lives of many social animals, to our knowledge there have been no studies on decisions about the timing of return to sleeping sites. Here we analysed longterm, low-resolution (ca. $1 \mathrm{fix} / 15 \mathrm{~min}$ ) GPS (global positioning system) data of meerkat groups combined with high-resolution ( $1 \mathrm{fix} / \mathrm{s}$ ) GPS data and continuous audio recordings on specific individuals, collected over a short period of time. Specifically, we investigated the ranging behaviour of wild meerkat groups to identify (1) how group movements are coordinated, (2) whether the dominant individuals or all group members control the time of the return, and what factors affect the (3) timing, (4) speed and (5) directness of the return.

\section{METHODS}

\section{Study Site and Population}

For this study, we used long-term data collected by the Kalahari Meerkat Project over the 13 years from 2002 to 2015 on more than 60 groups, as well as acoustic and high-resolution tracking data collected between January and March 2015 on six meerkat groups. The project is situated at the Kuruman River Reserve, in the Northern Cape of South Africa $\left(26^{\circ} 58^{\prime} S, 21^{\circ} 49^{\prime} E\right)$. Details on the habitat and population are provided elsewhere (Clutton-Brock et al., 1998). All animals in the population could be identified through individual dye mark combinations (Jordan, Cherry, \& Manser, 2007) and were habituated to close human handling and observation within less than $1 \mathrm{~m}$.

\section{Data Collection}

\section{Long-term data}

Data on each group, ranging from two to 49 individuals, were collected on approximately 3 days per week in the morning, the evening or both. Morning observation sessions started at dawn and ended after roughly $3 \mathrm{~h}$ of foraging. The evening observation session started when meerkats resumed foraging after resting during the hottest hours of the day, and ended after $1.5-2 \mathrm{~h}$, when the group returned to their sleeping burrow at sunset. The location of 
the morning and evening sleeping burrow, the number of individuals present in the group and the presence of pups (at the sleeping burrow or joining the foraging group) were recorded during each session. Furthermore, the observers following the group during each session took a GPS fix from the centre of the group every 15 min (accuracy: 95\% of fixes within $5 \mathrm{~m}$; eTrex $\mathrm{H}$, Garmin International Inc., Olathe, KS, U.S.A.). Each meerkat was trained to climb onto electronic balances for a small reward of boiled egg or water at the start and end of the morning sessions as well as at the end of the evening sessions. This allowed us to calculate the difference between an individual's evening and morning weight providing a measure of an individual's daily foraging success.

\section{Acoustic data and high-resolution tracking data}

Data were collected between January and March 2015 on six meerkat groups, with group sizes ranging from nine to 16 individuals (mean $\pm \mathrm{SD}=12.2 \pm 2.9$ ), during 17 evening sessions (described above), with two to three sessions per group and at least 3 days between visits to a group. The vocalizations and positions of four individuals (the dominant pair and two subordinates) were recorded by each of four observers. Vocalizations were recorded using a directional microphone (Sennheiser ME66 with K6 powering module) connected to a recorder (Marantz PMD660, sampling frequency $44.1 \mathrm{kHz}, 16 \mathrm{bit}$ ) $0.3-1.5 \mathrm{~m}$ from the individual. The position of each followed individual was inferred through the location of each observer, carrying a small device $(22 \mathrm{~g}, \mathrm{CCD}$ ltd) that recorded the GPS track at $1 \mathrm{~Hz}$ frequency (accuracy: $99 \%$ of fixes within $5 \mathrm{~m}, 82 \%$ of fixes within $2 \mathrm{~m}$ ) and sound continuously (sampling frequency $48 \mathrm{kHz}$, saved in mp3: bitrate $=160 \mathrm{kBit} / \mathrm{s}$ ), subsequently called GPS/ audio unit. Up to four additional meerkats (mean $\pm S D=1.6 \pm 1.4$ individuals per group) were fitted with a GPS/audio collar before the start of each session. One person distracted the meerkat with water from a water bottle and another person attached the collar to the outstretched neck of the drinking meerkat. The length of the collar was adjusted in advance by measuring the neck of the meerkat during earlier sessions, to reduce the handling time for each individual. If a meerkat shied away from the collar, we did not attach it to the animal in question during this session.

\section{Ethical Note}

All data collection adhered to ASAB/ABS guidelines. All research was conducted under the permission of the ethical committee of Pretoria University (Permit number: EC011-10) and the Northern Cape Conservation Service, (FAUNA 1020/2016), South Africa.

\section{Data Analysis}

\section{Long-term data}

For the analysis of long-term data, we only included groups that had been observed in both the morning and evening for a minimum of 10 times. This left us with 34 groups (group size: mean \pm $\mathrm{SD}=14.89 \pm 7.01$ ) over the full study period. For the remaining data, we calculated the distance between GPS fixes as well as the distance between each fix and the evening sleeping burrow, using the packages 'move' (Kranstauber \& Smolla, 2016) and 'geosphere' (Segev, Kigel, Lubin, \& Tielbörger, 2015) in R (version 3.3.0.; R Core Team, 2016). From the distance between each GPS fix we calculated the total length of the foraging route throughout the whole day, standardized by the total time the group spent foraging. From each GPS fix in the afternoon session we calculated the distance to the evening sleeping burrow as well as the speed of the group during the 15 min time intervals between each GPS fix and from that the change in speed from one time interval to the next. If the time between the last GPS point before arrival at the sleeping burrow and the time of arrival was shorter than 10 min we excluded the distance information of this time step from the analysis. We determined the mean daily weight gain of the group by first calculating the difference in weight between the morning and the evening session for each individual, and then taking the mean for the whole group. Furthermore, we calculated the mean weight gain of the subordinate individuals as well as the weight gain of the dominant female and the dominant male of each group. To investigate the influence of pups (defined as dependent individuals up to the age of 90 days) on the change in speed during as well as on the timing of the return, we divided the presence of pups into three subcategories: (1) no pups present, (2) pups at the sleeping burrow and (3) pups foraging with the group. Finally, we calculated the time of sunset for each session, as meerkats generally return to their sleeping burrows around this time.

\section{Acoustic data and high-resolution tracking data}

For the analysis of the acoustic data, we excluded three sessions due to the meerkats returning early because of stormy weather or due to an intergroup interaction during the session. For the other 14 sessions, we calculated the centroid of the group (mean $\mathrm{x}, \mathrm{y}$ location of all observers and additional meerkats with GPS/audio units) as well as the time until sunset for each time step $(1 \mathrm{~Hz})$. We binned the vocalization data into 5 min time intervals, from the end of a session until the start of the next and in relation to the time of sunset. For each bin, we calculated the mean per capita call rates for both move and lead calls. We further calculated the call rate for the dominant female and the dominant male, as well as the mean call rate of subordinate meerkats. For the same time bins we scored the presence and absence of calls, which we used for the statistical analysis. Furthermore, we calculated the distance of the group's centroid to the sleeping burrow at the start of each of the time bins.

\section{Statistical Analysis}

\section{Acoustic data and high-resolution tracking data}

Statistical analysis was carried out using $\mathrm{R}$ (version 3.3.0.). To investigate whether move calls and lead calls were more likely to be produced when the group was far away from the burrow or when it was getting late, we fitted two generalized linear mixed-effects models (GLMM; Bates, Maechler, Bolker, \& Walker, 2015) with the presence/absence of either move calls or lead calls as the response variable. As the data were highly zero inflated, we used presence/ absence of calls as the response variable rather than absolute number, and assumed a binomial error distribution. This solved any problems with overdispersion (overdispersion test (Scrucca, 2004): observed variance/theoretical variance $=0.49$, statistic $=139.43$, $P=1$ ). We included distance to the sleeping burrow, time until sunset, and the interaction between these two predictors as fixed effects and controlled for the session nested in group by fitting them in the random term. The explanatory variables were checked for collinearity and z-transformed by first calculating the mean and standard deviation for each variable and then for each variable subtracting the mean and dividing by the standard deviation. We found no significant autocorrelation in the call rates of either move or lead calls. To assess the importance of each explanatory variable, we used multimodel inference, fitting models containing all possible combinations of predictors (using the dredge function in the MuMIn package in R), and then computing their weighted Akaike information criterion (AIC) scores using the importance function. All models were ranked based on their corrected AICc and we present all models within 2 delta AICc of the top-ranked model. Models within 2 AICc units of the best model were also used to calculate averaged effect sizes (standardized on two standard 
deviations following Gelman (2008)). We used a log likelihood ratio test to assess the significance of each of the fixed effects in the topranked model. The same process was used for all models described below and therefore is not described in detail again.

To determine whether dominant individuals called more than subordinate individuals we subset the data and included only time bins when at least one call (either move or lead call) was produced. We then log transformed the call rate and fitted a linear mixedeffects model (LMM) with the log transformed call rate as response variable, the dominance status and sex of an individual, namely the dominant female, dominant male or subordinate female, subordinate male, as explanatory variable and the session nested in group as random effects. Again, we found no collinearity between the explanatory variables or autocorrelation in any of the models. We used multimodel inference to assess the importance of each explanatory variable.

\section{Long-term movement data}

To investigate whether the time of return was affected by intrinsic or extrinsic factors as well as whether the decision on the timing was a shared or a unshared decision, we fitted a LMM with the time of return, i.e. the remaining time until sunset, as response variable and after checking for collinearity, the group size, the total length of the daily foraging route, as well as the interaction between route length and the presence of pups at the sleeping burrow and finally the interaction between the mean weight gain of the subordinate group members and the dominant female's and dominant male's weight gain, respectively, as explanatory variables, with group as random term. As described above we used multimodel inference to infer the relative importance of the different predictors, and used model averaging to estimate the strength and direction of each effect.

To test whether the mean speed of a meerkat group depended on extrinsic factors such as the length of the foraging route, the distance to the sleeping burrow, the group size and the remaining time until dusk or on the intrinsic state of meerkats indicated by their foraging success, we fitted an LMM with mean speed as the response variable and the number of nonpup group members, the mean weight gain, the interaction between the presence of pups at the sleeping burrow and the length of the daily foraging route, as well as the interaction between the distance to the sleeping burrow and the time left until sunset, as explanatory variables. These two interaction terms were added to capture the likely change in the length of the route when pups are present in the group as well as the urgency, namely being far away from the sleeping burrow and late. We added the session nested within the group as random factor to account for multiple measurements within each session. Explanatory variables were checked for collinearity and z-transformed prior to model fitting, again by first calculating the mean and standard deviation for each variable and then for each variable subtracting the mean and dividing by the standard deviation. Once more we used multimodel inference to assess the importance of each explanatory variable.

To investigate whether the trajectories of meerkat groups become more directed depending on time until sunset and distance from the sleeping burrow, we first extracted the turning angles along each group trajectory, defined as the angle between consecutive group direction vectors. Turning angles close to 0 are associated with more directed paths, whereas turning angles that deviate from 0 (in either direction) represent more tortuous paths. To visualize how directness varied with time until sunset and distance from the sleeping burrow, we binned the data into 10 bins along each dimension (time and distance), with bin boundaries set by the $10 \%$ quantiles of the distributions of time until sunset and distance from burrow, respectively. Next, we summarized the directness by calculating the circular variance of the turning angles for GPS data within each bin and subtracting this value from 1. Because turning angle distributions are centred around zero, this measure quantifies how much the turning angles deviate from 0 , with low values of directness indicating tortuous paths and high values indicating straight paths. Finally, we fitted a linear model with the circular variance as the response variable and the interaction between the binned distance to the sleeping burrow and the binned time until sunset as the explanatory variables. The explanatory variables were checked for collinearity.

\section{RESULTS}

\section{Acoustic Data}

Move calls were significantly more likely to be produced as the time until sunset decreased (estimate $\pm \mathrm{SE}=0.34 \pm 0.16$, log likelihood ratio test: $\chi^{2}=7.69, P=0.02, N=283$; Fig. 1). However, while the fitted model coefficients suggested slight trends (Fig. 2a), the probability of producing move calls did not change significantly based on the group's distance to the sleeping burrow (estimate $\pm \mathrm{SE}=0.13 \pm 0.21$, log likelihood ratio test: $\chi^{2}=3.24$, $P=0.20, N=283$ ) or the interaction between the time to sunset and the distance to the sleeping burrow (estimate $\pm \mathrm{SE}=0.06 \pm 0.12$, log likelihood ratio test: $\chi^{2}=2.16$, $P=0.14, N=283$; Fig. 2a). Lead calls, on the other hand, were increasingly likely to be produced based on the interaction between the remaining time until sunset and the distance to the sleeping burrow, with lead call probability increasing when meerkats were late in the foraging session and still far from their sleeping burrow (estimate $\pm S E=0.63 \pm 0.22$, log likelihood ratio test: $\chi^{2}=10.36$, $P=0.001, N=283$; Figs. 1 and $2 b$ ).

We found no overall difference in the number of move calls produced by individuals of different rank (log likelihood ratio test: $\chi^{2}=2.68, P=0.44, N=290$; Fig. 3a). Similarly, we found no overall difference in the number of lead calls produced by individuals of different rank (log likelihood ratio test: $\chi^{2}=5.32, P=0.15, N=84$; Fig. 3b).

\section{Long-term Data}

The speed and timing of meerkats' return to their sleeping site depended on the foraging success of the group as well as the

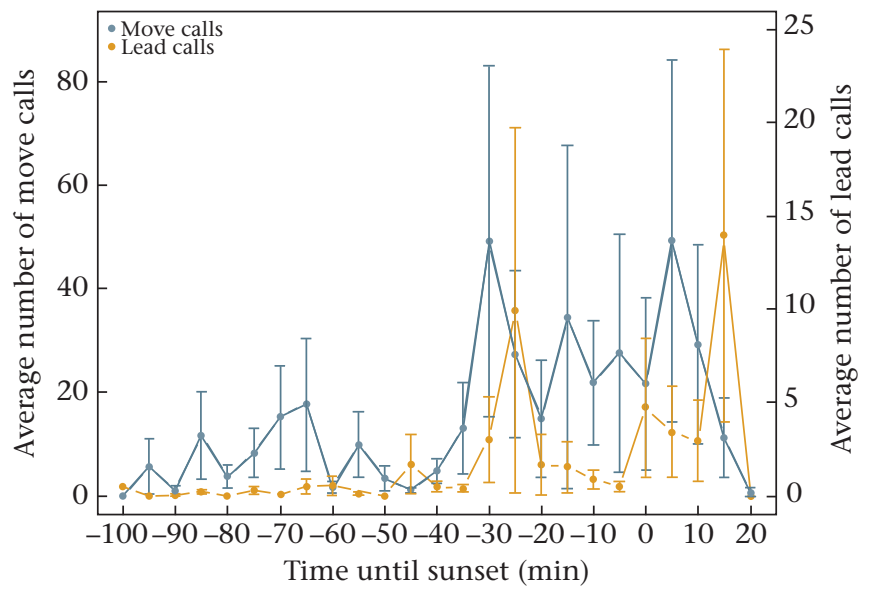

Figure 1. Mean move call rate and lead call rate per time bin (time until sunset). Times are binned into 5 min intervals, with negative values indicating times before sunset and positive values indicating times after sunset. Error bars indicate the standard error of call rates within each bin. 
(a)

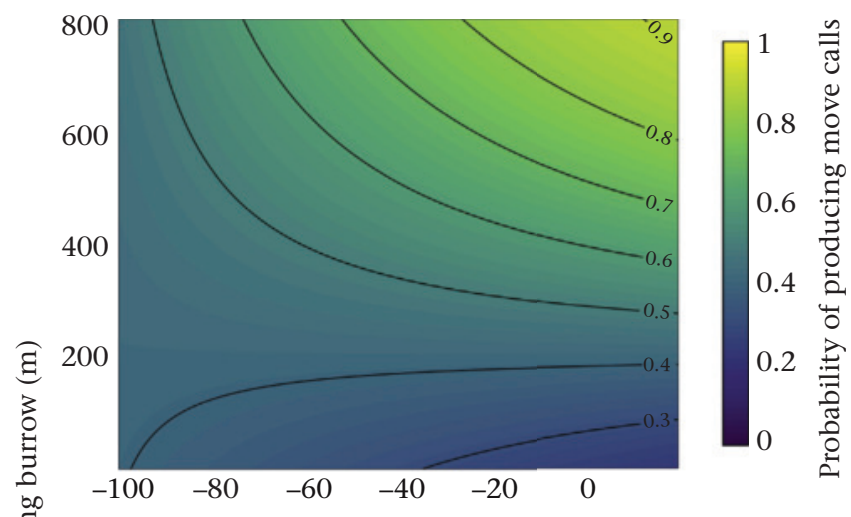

(b)

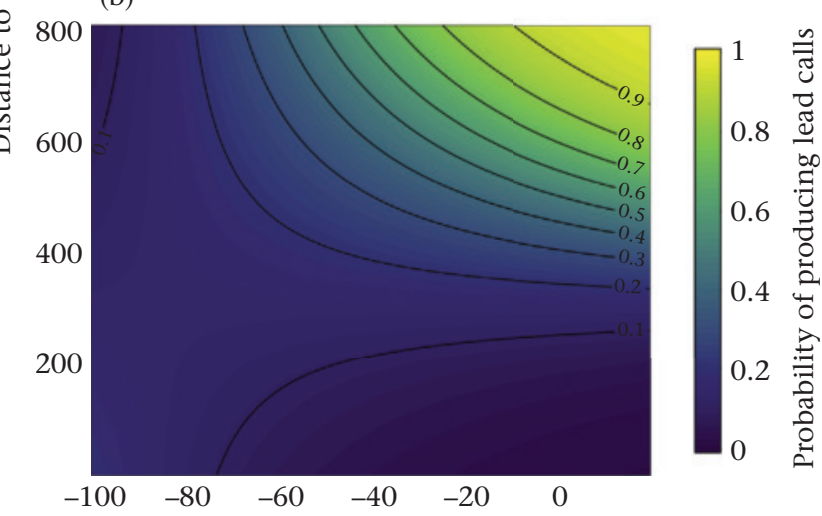

Time until sunset (min)

Figure 2. The probability of producing (a) move calls and (b) lead calls in relation to the distance to the sleeping burrow and the time left until sunset. The surface plot shows the predictions of the full model (LMM: including coefficients fitted for time until sunset, remaining distance to the burrow and the interaction between distance and time).

distance to the sleeping burrow and the remaining time until sunset (Fig. 4a). In particular, the mean speed of a group in the afternoon session was associated with the interaction between the distance to the evening sleeping burrow and the time until sunset (Appendix Fig. A1, Table A1), with groups increasing their speed when they were far away and sunset was imminent. Speed was also affected by foraging success, with groups moving at higher speed when the mean weight gain of the group was higher (Appendix Table A1). We found no significant interaction between the presence of pups and the length of the daily foraging route. Larger groups moved at greater speed than smaller groups (Appendix Table A1).

Meerkats also arrived significantly later at their sleeping burrow when the daily foraging route was long, when the group size was small, when pups were present, and when the mean weight gain of subordinate group members was low (Fig. 4b, Appendix Table A2). We found no significant effect of the dominant female's and dominant male's weight gain on the timing of the return (Appendix Table A2). The circular variance of turning angles was significantly affected by the time until sunset ( $\log$ likelihood ratio test: $F=18.54$, $P<0.001, N=100$ ) and the distance to the sleeping burrow (log likelihood ratio test: $F=8.99, P<0.001, N=100$ ), but not the interaction between the two (log likelihood ratio test: $F=0.04$, $P=0.85, N=100$ ), with groups becoming more directed the further they were from the burrow and the less time remaining until sunset (Fig. 5).

\section{DISCUSSION}

Here we investigated how the decision to return to the sleeping burrow in meerkats was coordinated, whether it was shared among group members, and what factors affected the timing, speed and directness of return. Meerkats used two call types when initiating the return to the sleeping site. We found that move calls were more likely to be produced as dusk approached (with no significant effect of the distance to the burrow), while the production of lead calls depended on the interaction between the time until sunset and the distance to the burrow, with the highest lead call rates occurring when dusk was imminent and the meerkats were far from their sleeping burrow. Call rate did not depend on the rank or sex of an individual, with subordinate individuals producing similar numbers of move and lead calls as the dominant pair, suggesting that the decision to return is not monopolized by dominant individuals. The speed of the group at the return changed depending on the distance of the group to the sleeping burrow, the time until sunset, the mean daily weight gain of a group, group size, the length of the foraging route and the presence of pups. This shows that the type of return (slow versus fast) is affected by both intrinsic and extrinsic factors. Furthermore, groups moving faster the further they were from the burrow and the later it was indicates that the type of return is based on the urgency for the group to return. The movement of meerkat groups became more directed (smaller turning angles) the further away they were from their burrow, and the less time remaining until nightfall. Meerkats returned later to their burrow when group size was small, when pups were present (either at the sleeping burrow or foraging with the group), when their daily foraging route was long and when the mean weight gain of subordinates was low. The weight gain of the dominant female and the dominant male had lower importance (based on multimodel inference) in affecting the timing of the return compared to the weight gain of subordinates. This is in line with our hypothesis that the return time is affected by the intrinsic state of an individual, measured as its daily foraging success. In addition, it suggests that the decision to return is shared among multiple individuals rather than being monopolized by dominant individuals.

The dominant pair are the social leaders of the group and it has previously been shown that the dominant female is the most likely individual to initiate group departure from the burrow when her energetic demands are increased due to pregnancy or lactation periods (Turbé, 2006). In contrast, our results are more in line with the hypothesis that the decision to return to the burrow is shared. If either of the dominant individuals controlled the decision to return, we would expect their weight gain to be more important in predicting the group's return time than the weight gain of subordinates. However, we found that the mean weight gain of subordinates was more important in the model predictions, with the group returning later when the weight gain of subordinate group members was low. In addition, the finding that subordinate meerkats gave similar numbers of move and lead calls as the dominant pair supports the hypothesis that the decision to return is shared. However, this does not exclude the possibility that group members might be more likely to follow calls given by dominant rather than subordinate individuals. Furthermore, the current study does not test whether individuals may still vary in their propensity to give move and lead calls or to be followed independent of dominance. Experiments will be necessary to confirm the hypothesis of shared decision making. For example, move and lead calls from different individuals within the group should be played and the movement response of the group recorded.

For many diurnal species, predation risk increases with the approach of dusk, as predator detection decreases (Lima \& Dill, 

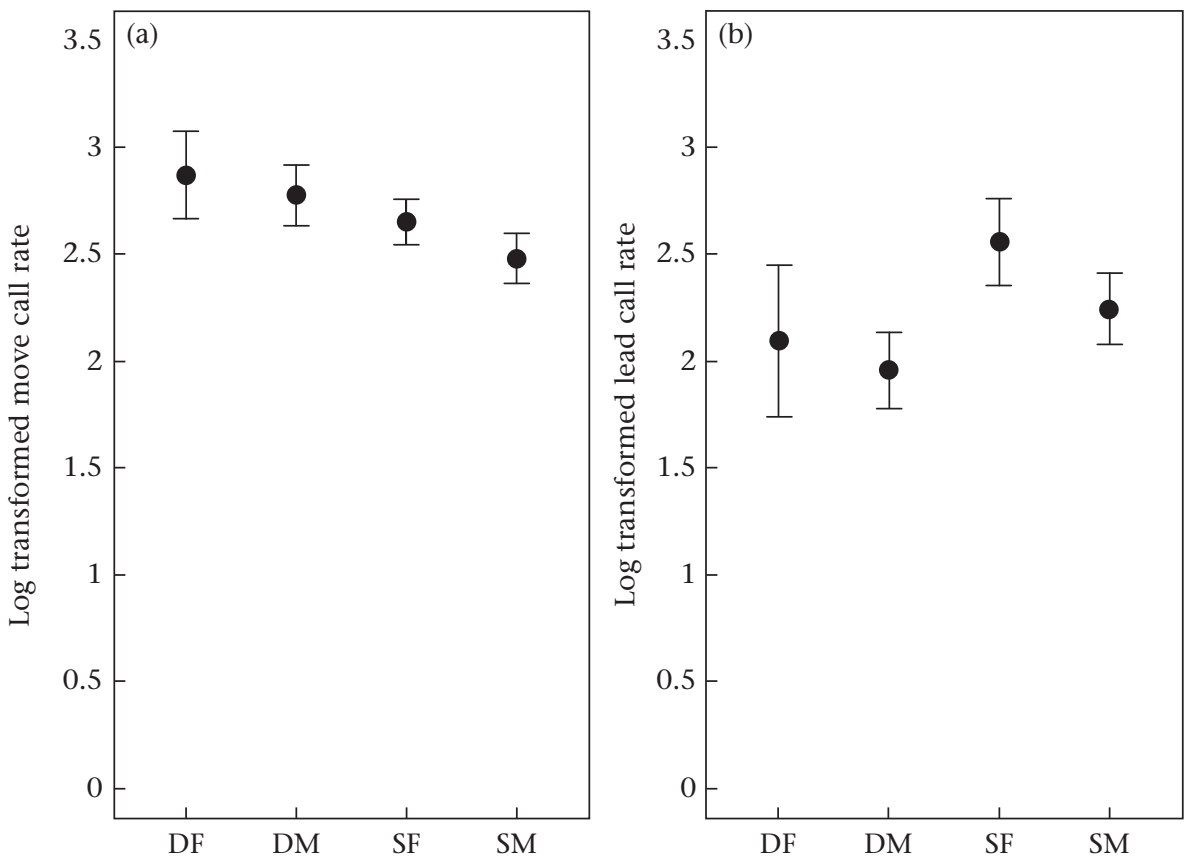

Figure 3. Mean (point) and standard error (error bars) of the log transformed number of (a) move calls and (b) lead calls per 5 min time interval for each dominance and sex category. ( $\mathrm{DF}=$ dominant female; $\mathrm{DM}=$ dominant male; $\mathrm{SF}=$ subordinate female; $\mathrm{SM}=$ subordinate male).

1990; Rand, Bridarolli, Dries, \& Ryan, 1997) and dropping temperatures after sunset can lead to increased heat loss for each individual (Terrien, Perret, \& Aujard, 2011). These factors increase the costs for each individual substantially as night falls, probably leading to an increase in the urgency for the group to return to its sleeping site. This suggests that the speed of return and the directness of a group are urgency based, with groups increasing their speed and moving in a more directed fashion shortly before sunset and when they are far away from their burrow. The use of move and lead calls may also be based on urgency, as the probability of producing move calls depended only on the remaining time until sunset, but the probability of producing lead calls depended on both the time and the distance to the burrow. Move calls have been shown to be used during quorum decisions (Bousquet et al., 2011) while lead calls were shown to be followed when produced by a single individual (Turbé, 2006), indicating a potential change from a shared decision to an unshared decision (although not necessarily led by the dominant individual) with an increase in urgency. This would be similar to house-hunting ants, shown to adjust their decision time by decreasing their quorum threshold when in an urgent situation (Franks et al., 2003). However, to fully understand how the decision-making process of meerkats is affected by urgency, controlled experiments will be needed.

Our results show that the type and the timing of the return depend on the intrinsic state of group members, here measured through daily weight gain. When the foraging success of the group is low (low mean daily weight gain), individuals might compensate by increasing the time searching for food and returning later, thereby moving faster when returning at the last possible moment. This is also consistent with the idea that when the daily foraging route is very long, groups must move at greater speeds in order to return to the burrow before dark. Similarly, when pups are at the burrow or join the foraging group, the energetic demands of group members increase (Brotherton et al., 2001; Clutton-Brock,
Brotherton, et al., 2001; Clutton-Brock, Russell, et al., 2001; Russell et al., 2002) and individuals might therefore prolong their foraging time to satisfy these energetic demands. In general, meerkats do not change their sleeping site while they have pups at their burrow (Clutton-Brock et al., 1998), and this will affect their foraging success and their choice of foraging routes, for example groups might stay closer to the babysitting burrow while foraging patches are still available and might travel further when foraging patches are depleted. While bigger groups seemed to generally move faster, smaller groups returned later. This could be due to the increased need for each individual to be vigilant in smaller groups (Clutton-Brock et al., 1999) and the ensuing reduction in time spent foraging.

The timing and the speed of return also depended on extrinsic factors such as the distance to the sleeping burrow and the remaining daylight. This is to be expected, as when the group is far from a burrow and it is getting dark, it needs to cover larger distances in a short amount of time. If the decision about the timing of return were solely based on the remaining distance to the burrow, we would expect meerkats to return at the same speed independent of distance to their burrow. This is because the decision to return would be taken earlier when the group is far away and later when the group is very close. It follows that the actual decision on the timing of the return might depend mainly on intrinsic states, for example level of satiation related to the foraging success, while the type of return, that is, slow versus fast, might be based on extrinsic factors, such as the remaining daylight time and the distance to the burrow.

In conclusion, our results provide evidence that the decision about the timing of the return is shared among multiple group members, and suggest that the speed of the group and the use of vocalizations are based on urgency. This raises the possibility that the underlying decision process changes from shared to unshared depending on time constraints, as suggested by the fact that lead calls were given more often when the group was far from its 


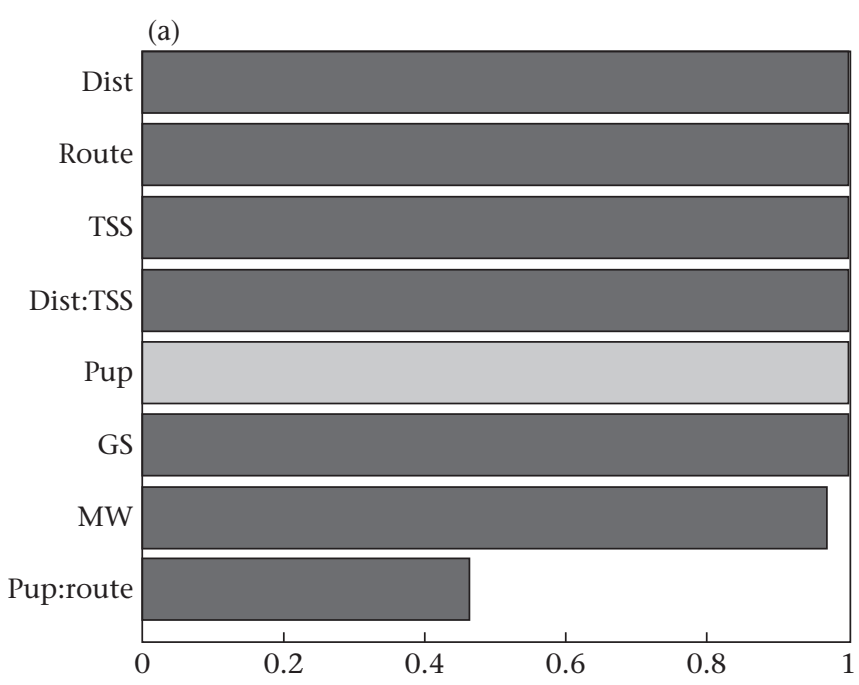

(b)

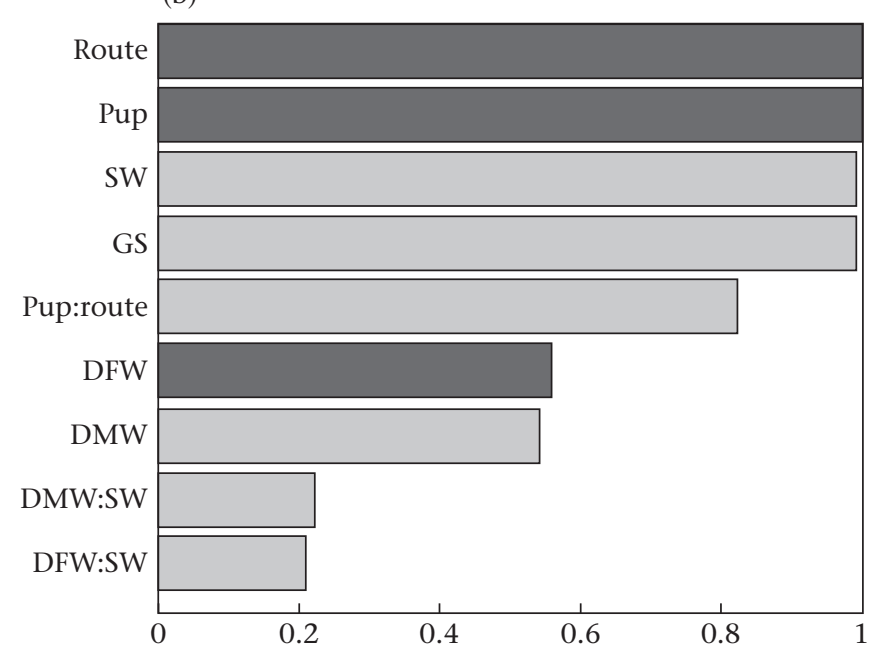

Figure 4. Importance scores from the multimodel inference on (a) the speed of the return to the burrow and (b) the time of the return to the burrow. Light grey bars represent a negative coefficient and dark grey a positive coefficient (see Appendix Tables A1 and A2). The explanatory variables are Dist (distance to the sleeping burrow), route (the total length of the daily foraging route), TSS (time remaining until sunset), the interaction between Dist and TSS, Pup (presence/absence of pups at the burrow and pups foraging with the group), GS (group size), MW (mean weight gain of the group), the interaction between Pup and Route, SW (the mean weight gain of subordinate group members), DFW (the dominant female's weight gain), DMW (the dominant male's weight gain), and the interaction between DMW and DW and DFW and SW, respectively.

burrow and when dark was falling. Furthermore, we have shown that the timing of the return to the burrow was dependent on the internal state of group members, whereas the travel speed at the return was also influenced by external factors. While we find parallels to initiation processes from resting to foraging, the decisionmaking process in the evening, which initiates a change from foraging to resting, is more likely to be shared, whereas decisions from resting to foraging have been shown to be unshared in meerkats (Turbé, 2006). Our study highlights the importance of time constraints during decision-making processes and their impact on the level of decision sharing in natural systems. Fully understanding how animal groups make consensus decisions will require additional studies of the initiation of changes in group activities under time constraints, including experimental manipulations, across different contexts.

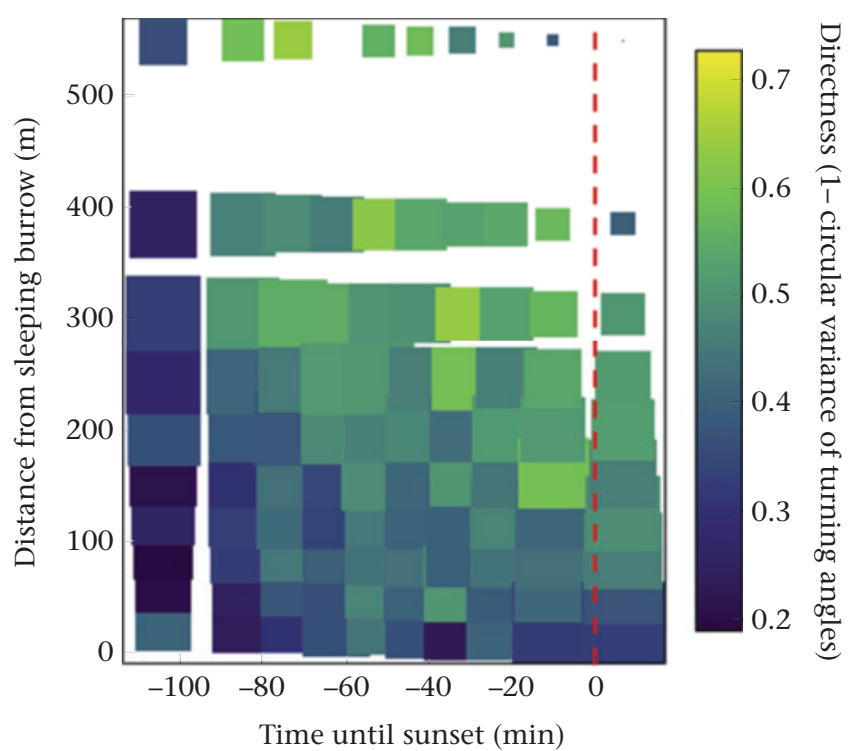

Figure 5. Directness (i.e. 1 - circular variance in turning angles) of meerkat groups as a function of time until sunset and distance from the sleeping burrow. Bins represent $10 \%$ quantiles of times and distances, respectively, and point sizes indicate the amount of data in each bin (log scale). The points are plotted at the median of each bin, e.g. the last distance bin ranges from approximately $430 \mathrm{~m}$ to $1000 \mathrm{~m}$ to the sleeping burrow, with the median at $540 \mathrm{~m}$. The red dashed line indicates the time of sunset. Negative values give times before sunset and positive numbers times after sunset.

\section{COMPETING INTERESTS}

We have no competing interests.

\section{AUTHOR CONTRIBUTIONS}

The long-term data were collected by the Kalahari Meerkat Project organized by T.H.C-B. and M.M., and the vocal and highresolution GPS data by G.G. and M.M. The data were analysed by G.G. and A.S.P. and the article was written by G.G., A.S.P., T.H.C-B. and M.M. All authors gave final approval for publication.

\section{Acknowledgments}

We thank the Kalahari Research Trust for permission to work at the Kalahari Meerkat Project (KMP) and the neighbouring farmers to work on their land. We also thank Dave Gaynor for the organization of the field site and his input on the field work, and the managers and volunteers of the KMP for maintaining the habituation and basic data collection on the meerkats. Further, we thank Bruce Boatman, Pauline Toni and Denise Camenisch for their assistance in collecting the vocal and high-resolution GPS data, and two anonymous referees for comments on the manuscript. This study was funded by the Swiss National Science Foundation (Grant No. PDFMP3_141768) to M.M. The long-term field site KMP was financed by Cambridge University and Zurich University. This paper has relied on data collected and maintained by the KMP, which has been supported by the European Research Council (Research Grant No. 294494 to T.H.C-B. since 1 July 2012), the University of Zurich and the Mammal Research Institute of the University of Pretoria.

\section{References}

Bates, D., Maechler, M., Bolker, B., \& Walker, S. (2015). Fitting linear mixed-effects models using lme4. Journal of Statistical Software, 67(1), 1-48. https://cran.rproject.org/web/packages/lme4/index.html. 
Boinski, S. (1993). Vocal coordination of troop movement among white-faced capuchin monkeys, Cebus capucinus. American Journal of Primatology, 30(2), 85-100. http://dx.doi.org/10.1002/ajp.1350300202.

Bousquet, C. A. H., Sumpter, D. J. T., \& Manser, M. B. (2011). Moving calls: A vocal mechanism underlying quorum decisions in cohesive groups. Proceedings of the Royal Society B: Biological Sciences, 278(1711), 1482-1488. http://dx.doi.org/ 10.1098/rspb.2010.1739.

Brotherton, P. N. M., Clutton-Brock, T. H., O'Riain, M. J., Gaynor, D., Sharpe, L. Kansky, R., et al. (2001). Offspring food allocation by parents and helpers in a cooperative mammal. Behavioral Ecology, 12(5), 590-599. http://dx.doi.org/ 10.1093/beheco/12.5.590.

Chittka, L., Skorupski, P., \& Raine, N. E. (2009). Speed-accuracy tradeoffs in anima decision making. Trends in Ecology \& Evolution, 24(7), 400-407. http:// dx.doi.org/10.1016/j.tree.2009.02.010.

Clutton-Brock, T. H., Brotherton, P. N. M., O'Riain, M. J., Griffin, A. S., Gaynor, D. Kansky, R., et al. (2001a). Contributions to cooperative rearing in meerkats. Animal Behaviour, 61, 705-710. http://dx.doi.org/10.1006/anbe.2000.1631.

Clutton-Brock, T. H., Grynor, D., Kansky, R., MacColl, A. D. C., Macllrath, G. Chadwick, P., et al. (1998). Costs of cooperative behavior in suricates (Suricata suricatta). Proceedings of the Royal Society B: Biological Sciences, 265(1392), 185-190.

Clutton-Brock, T. H., O'Riain, M. J., Brotherton, P. N. M., Gaynor, D., Kansky, R. Griffin, A. S., et al. (1999). Selfish sentinels in cooperative mammals. Science, 284(5420), 1640-1644. http://dx.doi.org/10.1126/science.284.5420.1640.

Clutton-Brock, T. H., Russell, A. F., Sharpe, L. L., Brotherton, P. N. M., Mcllrath, G. M., White, S., et al. (2001b). Effects of helpers on juvenile development and survival in meerkats. Science, 293(5539), 2446-2449. http://dx.doi.org/10.1126/ science.1061274.

Conradt, L. (2011). When it pays to share decisions. Nature, 471, 40-41.

Conradt, L., \& Roper, T. J. (2003). Group decision-making in animals. Nature, 421(6919), 155-158. http://dx.doi.org/10.1038/nature01294.

Conradt, L., \& Roper, T. J. (2005). Consensus decision making in animals. Trends in Ecology \& Evolution, 20(8), 449-456. http://dx.doi.org/10.1016/ j.tree.2005.05.008.

Conradt, L., \& Roper, T. J. (2007). Democracy in animals: The evolution of shared group decisions. Proceedings of the Royal Society B: Biological Sciences, 274(1623), 2317-2326. http://dx.doi.org/10.1098/rspb.2007.0186.

Conradt, L., \& Roper, T. J. (2009). Conflicts of interest and the evolution of decision sharing. Philosophical Transactions of the Royal Society B: Biological Sciences, 364(1518), 807-819. http://dx.doi.org/10.1098/rstb.2008.0257.

Dunbar, R. I. M., \& Dunbar, P. (1988). Maternal time budgets of gelada baboons. Animal Behaviour, 36, 970-980. http://dx.doi.org/10.1016/s0003-3472(88) 80055-1.

Fischhoff, I. R., Sundaresan, S. R., Cordingley, J., Larkin, H. M., Sellier, M. J., \& Rubenstein, D. I. (2007). Social relationships and reproductive state influence leadership roles in movements of plains zebra, Equus burchellii. Animal Behaviour, 73, 825-831. http://dx.doi.org/10.1016/j.anbehav.2006.10.012.

Fleischmann, D., \& Kerth, G. (2014). Roosting behavior and group decision making in 2 syntopic bat species with fission-fusion societies. Behavioral Ecology, 25(5), 1240-1247. http://dx.doi.org/10.1093/beheco/aru117.

Franks, N. R., Dechaume-Moncharmont, F. X., Hanmore, E., \& Reynolds, J. K. (2009) Speed versus accuracy in decision-making ants: Expediting politics and policy implementation. Philosophical Transactions of the Royal Society B: Biological Sciences, 364(1518), 845-852. http://dx.doi.org/10.1098/rstb.2008.0224.

Franks, N. R., Dornhaus, A., Fitzsimmons, J. P., \& Stevens, M. (2003). Speed versus accuracy in collective decision making. Proceedings of the Royal Society B: Biological Sciences, 270(1532), 2457-2463. http://dx.doi.org/10.1098/ rspb.2003.2527.

Furrer, R. D., Kunc, H. P. \& Manser, M. B. (2012). Variable initiators of group departure in a cooperative breeder: The influence of sex, age, state and foraging success. Animal Behaviour, 84(1), 205-212.

Gelman, A. (2008). Growth rates in epidemic models: Application to a model for HIV/AIDS progression. Statistics in Medicine, 27, 2865-2873. http://dx.doi.org/ $10.1002 / \mathrm{sim}$.

Huang, C. M., Wei, F. W., Li, M., Li, Y. B., \& Sun, R. Y. (2003). Sleeping cave selection, activity pattern and time budget of white-headed langurs. International Journal of Primatology, 24(4), 813-824. http://dx.doi.org/10.1023/a:1024628822271.

Jordan, N. R., Cherry, M. I., \& Manser, M. B. (2007). Latrine distribution and pattern of use by wild meerkats: Implications for territory and mate defense. Animal Behaviour, 73, 613-622.

Kalahari Meerkat Project (2017) [Long-term data.] Unpublished raw data.

King, A. J., Douglas, C. M. S., Huchard, E., Isaac, N. J. B., \& Cowlishaw, G. (2008). Dominance and affiliation mediate despotism in a social primate. Current Biology, 18(23), 1833-1838. http://dx.doi.org/10.1016/j.cub.2008.10.048.

King, A. J., \& Sueur, C. (2011). Where next? Group coordination and collective decision making by primates. International Journal of Primatology, 32(6) 1245-1267. http://dx.doi.org/10.1007/s10764-011-9526-7.
Kranstauber, B., \& Smolla, M. (2016). Move: visualizing and analyzing animal track data. $R$ package version 1.6.541. https://cran.r-project.org/web/packages/move/ index.html.

Krause, J., \& Ruxton, G. D. (2002). Living in groups. Oxford, U.K.: Oxford University Press.

Lima, S. L., \& Dill, L. M. (1990). Behavioral decisions made under the risk of predation - a review and prospectus. Canadian Journal of Zoology, 68(4), 619-640. http://dx.doi.org/10.1139/z90-092.

R Core Team. (2016). R: A language and environment for statistical computing. Vienna, Austria: R Foundation for Statistical Computing. https://cran.r-project.org/.

Rand, A. S., Bridarolli, M. E., Dries, L., \& Ryan, M. J. (1997). Light levels influence female choice in Tungara frogs: Predation risk assessment? Copeia, 2, 447-450. http://dx.doi.org/10.2307/1447770.

Russell, A. F., Clutton-Brock, T. H., Brotherton, P. N. M., Sharpe, L. L., Mcllrath, G. M. Dalerum, F. D., et al. (2002). Factors affecting pup growth and survival in cooperatively breeding meerkats Suricata suricatta. Journal of Animal Ecology, 71(4), 700-709. http://dx.doi.org/10.1046/j.1365-2656.2002.00636.x.

Scrucca, L. (2004). qcc: An R package for quality control charting and statistical process control. $R$ News, 4(1), 11-17. https://cran.r-project.org/web/packages/ qcc/index.html.

Segev, U., Kigel, J., Lubin, Y., \& Tielbörger, K. (2015). Ant abundance along a productivity gradient: Addressing two conflicting hypotheses. PLoS One, 10(7) 1-17. http://dx.doi.org/10.1371/journal.pone.0131314.

Sumpter, D. J. T., \& Pratt, S. C. (2009). Quorum responses and consensus decision making. Philosophical Transactions of the Royal Society B: Biological Sciences, 364(1518), 743-753. http://dx.doi.org/10.1098/rstb.2008.0204.

Terrien, J., Perret, M., \& Aujard, F. (2011). Behavioral thermoregulation in mammals: A review. Frontiers in Bioscience-Landmark, 16, 1428-1444. http://dx.doi.org/ $10.2741 / 3797$

Turbé, A. (2006). Foraging decisions and space use in an asocial mammal, the meerkat (PhD thesis). Cambridge, U.K.: University of Cambridge.

Ward, A. J. W., Herbert-Read, J. E., Sumpter, D. J. T., \& Krause, J. (2011). Fast and accurate decisions through collective vigilance in fish shoals. Proceedings of the National Academy of Sciences of the United States of America, 108(6), 2312-2315. http://dx.doi.org/10.1073/pnas.1007102108.

Ward, A. J. W., Sumpter, D. J. T., Couzin, L. D., Hart, P. J. B., \& Krause, J. (2008). Quorum decision-making facilitates information transfer in fish shoals. Proceedings of the National Academy of Sciences of the United States of America, 105(19), 6948-6953. http://dx.doi.org/10.1073/pnas.0710344105.

\section{APPENDIX}

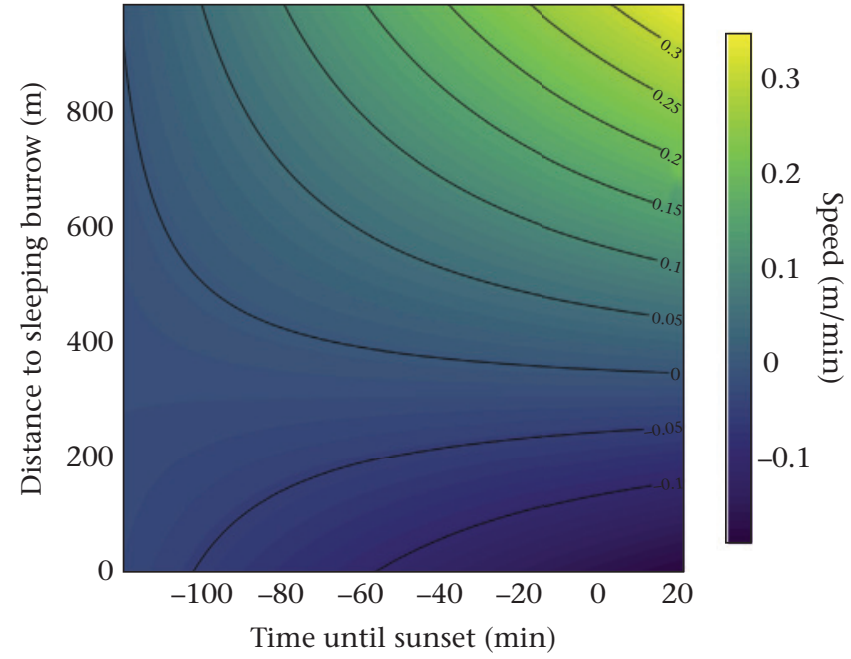

Figure A1. Mean speed of a meerkat group in relation to the distance to the sleeping burrow and the time until sunset. Negative numbers indicate times before sunset and positive numbers times after sunset and ' 0 ' indicates the time of sunset. The surface plot shows the predicted values based on the linear mixed-effects model (LMM). 
Table A1

Model summary statistics for the effects of different explanatory factors on mean speed of the group using data calculated from $N=1880$ sessions

\begin{tabular}{|c|c|c|c|c|c|c|c|c|c|c|c|c|c|c|c|c|}
\hline & Intercept & Dist & MW & GS & \multicolumn{2}{|l|}{ Pup } & Route & TSS & Dist*TSS & \multicolumn{2}{|c|}{ Pup*Route } & $d f$ & Log likelihood & AICc & $\Delta \mathrm{AICc}$ & $w$ \\
\hline Model 1 & 0.085 & 0.012 & 0.002 & 0.003 & + & & 0.024 & 0.027 & 0.015 & & & 12 & 12605.64 & -25187.2 & 0.00 & 0.53 \\
\hline Model 2 & 0.085 & 0.012 & 0.002 & 0.003 & + & & 0.024 & 0.027 & 0.015 & + & & 14 & 12607.38 & -25186.7 & 0.52 & 0.41 \\
\hline Model 3 & 0.085 & 0.012 & & 0.003 & + & & 0.024 & 0.027 & 0.015 & & & 11 & 12601.66 & -25181.3 & 5.94 & 0.03 \\
\hline Model 4 & 0.085 & 0.012 & & 0.003 & + & & 0.023 & 0.027 & 0.015 & + & & 13 & 12603.64 & -25181.2 & 5.99 & 0.03 \\
\hline Averaged parameters & & & & & & & & & & & & & & & & \\
\hline Estimate & 0.085 & 0.012 & 0.002 & 0.003 & -0.009 & -0.009 & 0.024 & 0.023 & 0.015 & 0.002 & 0.000 & & & & & \\
\hline Unconditional SE & 0.001 & 0.001 & 0.001 & 0.001 & 0.003 & 0.001 & 0.001 & 0.001 & 0.001 & 0.003 & 0.001 & & & & & \\
\hline Upper 95\% CI & 0.087 & 0.014 & 0.003 & 0.004 & -0.004 & -0.006 & 0.026 & 0.028 & 0.016 & 0.007 & 0.003 & & & & & \\
\hline Lower 95\% CI & 0.082 & 0.010 & 0.001 & 0.002 & -0.015 & -0.011 & 0.022 & 0.025 & 0.014 & -0.003 & -0.002 & & & & & \\
\hline Relative importance & & 1.00 & 0.97 & 1.00 & 1.00 & & 1.00 & 1.00 & 1.00 & 0.46 & & & & & & \\
\hline
\end{tabular}

The mean speed of the group was used as response variable in an LMM. Models within 2 AICc units of the best model are highlighted in bold and were used to calculate averaged effect sizes (standardized on two standard deviations following Gelman (2008)). Factors included are: Dist (distance to the sleeping burrow), MW (mean weight gain of the group), GS (group size), Pup (absence of pups, presence of pups at the burrow and pups foraging with the group), Route (the total length of the daily foraging route), TSS (time until sunset) and the interactions between Dist and TSS and Pup and Route, respectively. AIC: Akaike's information criterion; w: relative model weights.

Table A2

Model summary statistics for the effects of different explanatory factors on the return time calculated on data collected during $N=739$ sessions

\begin{tabular}{|c|c|c|c|c|c|c|c|c|c|c|c|c|c|c|c|c|c|}
\hline & Intercept & DFW & DMW & SW & GS & \multicolumn{2}{|l|}{ Pup } & Route & DFW*SW & DMW*SW & \multicolumn{2}{|c|}{ Pup*Route } & $d f$ & Log likelihood & AICc & $\Delta \mathrm{AICC}$ & $w$ \\
\hline Model 1 & -27.90 & & & -2.26 & -2.40 & + & & 4.00 & & & + & & 10 & -3146.60 & 6313.5 & 0.00 & 0.21 \\
\hline Model 2 & -27.84 & 0.42 & & -2.48 & -2.41 & + & & 4.01 & & & + & & 11 & -3145.81 & 6314.0 & 0.47 & 0.17 \\
\hline Model 3 & -27.90 & & -0.09 & -2.22 & -2.40 & + & & 4.00 & & & + & & 11 & -3145.98 & 6314.3 & 0.81 & 0.14 \\
\hline Model 4 & -27.83 & 0.46 & -0.17 & -2.41 & -2.41 & + & & 4.01 & & & + & & 12 & -3145.15 & 6314.7 & 1.22 & 0.12 \\
\hline Model 5 & -27.61 & & -0.06 & -2.23 & -2.41 & + & & 4.06 & & -0.51 & + & & 12 & -3145.24 & 6314.9 & 1.40 & 0.11 \\
\hline Model 6 & -27.67 & 0.36 & & -2.40 & -2.42 & + & & 4.01 & -0.36 & & + & & 12 & -3144.28 & 6315.0 & 1.48 & 0.10 \\
\hline Model 7 & -27.56 & 0.42 & -0.13 & -2.40 & -2.42 & + & & 4.07 & & -0.49 & + & & 13 & -3144.44 & 6315.4 & 1.87 & 0.08 \\
\hline Model 8 & -27.65 & 0.40 & -0.22 & -2.31 & -2.42 & + & & 4.01 & -0.37 & & & & 13 & -3144.60 & 6315.7 & 2.19 & 0.07 \\
\hline $\begin{array}{l}\ldots \\
\text { Averaged parameters }\end{array}$ & & & & & & & & & & & & & & & & & \\
\hline Estimate & -27.79 & 0.21 & -0.05 & -2.34 & -2.41 & 0.21 & 5.35 & 4.01 & -0.04 & -0.10 & -1.23 & -1.71 & & & & & \\
\hline Unconditional SE & 1.44 & 0.58 & 0.52 & 0.76 & 0.74 & 2.82 & 1.44 & 0.85 & 0.21 & 0.31 & 1.96 & 1.51 & & & & & \\
\hline Upper 95\% CI & -24.96 & 1.35 & 0.97 & -0.85 & -0.96 & 5.74 & 8.17 & 5.70 & 0.37 & 0.51 & 2.61 & 1.26 & & & & & \\
\hline Lower $95 \% \mathrm{CI}$ & -30.63 & -0.93 & -1.08 & -3.83 & -3.86 & -5.33 & 2.52 & 2.34 & -0.46 & -0.72 & -5.07 & -4.68 & & & & & \\
\hline Relative importance & & 0.56 & 0.54 & 0.99 & 0.99 & 1.00 & & 1.00 & 0.21 & 0.22 & 0.82 & & & & & & \\
\hline
\end{tabular}

The remaining time to sunset was used as response variable in an LMM. Models within 2 AICc units of the best model are highlighted in bold and were used to calculate averaged effect sizes (the estimate was standardized on two standard deviations following Gelman (2008)). Factors included are: DFW (the dominant female's weight gain), DMW (the dominant male's weight gain), SW (the mean weight gain of subordinate group members), GS (group size), Pup (absence of pups, presence of pups at the burrow and pups foraging with the group), Route (the total length of the daily foraging route), and the interaction between DFW and SW, DMW and SW and Pup and Route. AIC: Akaike's information criterion; $w$ : relative model weights. 